\title{
Confinement properties of high density impurity seeded ELMy H-mode discharges at low and high triangularity on JET
}

\author{
P Dumortier ${ }^{1,13}$, P Andrew ${ }^{2}$, G Bonheure ${ }^{1,13}$, R V Budny ${ }^{3}$, R Buttery $^{2}$, \\ M Charlet ${ }^{2}$, I Coffey ${ }^{2}$, M de Baar ${ }^{4,13}$, P C de Vries ${ }^{4,13}$, T Eich ${ }^{5,13}$, \\ D Hillis ${ }^{6}$, C Ingesson ${ }^{4,13}$, S Jachmich ${ }^{1,13}$, G Jackson ${ }^{7}$, A Kallenbach ${ }^{8}$, \\ H R Koslowski ${ }^{5,13}$, K D Lawson ${ }^{2}$, C Liu $^{9}$, G Maddison ${ }^{2}$, \\ A M Messiaen ${ }^{1,13}$, P Monier-Garbet $^{10}$, M Murakami $^{7}$, M F F Nave ${ }^{11}$, \\ J Ongena ${ }^{1,13}$, V Parail ${ }^{2}$, M E Puiatti ${ }^{12}$, J Rapp ${ }^{5,13}$, F Sartori ${ }^{2}$, M Stamp ${ }^{2}$, \\ J D Strachan ${ }^{3}$, W Suttrop ${ }^{8}$, G Telesca ${ }^{12}$, M Tokar ${ }^{5,13}$, B Unterberg ${ }^{5,13}$, \\ $M$ Valisa $^{12}$, M von Hellermann ${ }^{4}, B$ Weyssow $^{9}$ and contributors to the \\ EFDA-JET Workprogramme \\ ${ }^{1}$ Laboratory for Plasma Physics, Association Euratom-Belgian State, Koninklijke Militaire \\ School-Ecole Royale Militaire, Renaissancelaan, 30, avenue de la Renaissance, B-1000 \\ Brussels, Belgium \\ 2 EURATOM/UKAEA Fusion Association, Culham Science Center, Abingdon, Oxon OX14 \\ 3DB, UK \\ ${ }^{3}$ Princeton Plasma Physics Laboratory, Princeton University, NJ 08543, USA \\ ${ }^{4}$ FOM-Instituut voor Plasma fysica, EURATOM Association, Postbus 1207, NL-3430 BE \\ Nieuwegein, NL \\ ${ }^{5}$ Institut für Plasmaphysik, Forschungszentrum Jülich GmbH, EURATOM Association, \\ D-52425 Jülich, Germany \\ ${ }^{6}$ Oak Ridge National Laboratory, Oak Ridge, TN 37831, USA \\ 7 DIII-D National Fusion Facility, San Diego, CA 92186-5698, USA \\ ${ }^{8}$ Max-Planck-Institut für Plasmaphysik, IPP-EURATOM Assoziation, Boltzmann-Str.2, \\ D-85748 Garching, Germany \\ ${ }^{9}$ Service Physique Statistique et Plasmas, Association Euratom-Belgian State, Université Libre \\ de Bruxelles, Campus Plaine, CP 231, Bvd du Triomphe, B-1050 Brussels, Belgium \\ ${ }^{10}$ Association EURATOM/CEA CEA CADARACHE, DRFC, Bâtiment 513, 13108 \\ Saint-Paul-Lez-Durance, France \\ 11 Associação Euratom-IST, Centro de Fusão Nuclear, 1049-001 Lisbon, Portugal \\ 12 Consorzio RFX-Associazione Euratom-Enea sulla Fusione, Corso Stati Uniti 4, I-35127 \\ Padova, Italy
}

Received 1 July 2002

Published 27 August 2002

Online at stacks.iop.org/PPCF/44/1845

\begin{abstract}
The design value for ITER is based on operation at $n / n_{\mathrm{GW}}=0.85, \beta_{\mathrm{n}}=1.8$ and $\mathrm{H} 98(\mathrm{y}, 2)=1$. These values have been routinely achieved in JET in argon seeded ELMy H-mode discharges in different divertor configurations and with different triangularities. Two main scenarios are emerging from the experiments.
\end{abstract}

${ }^{13}$ Affiliations 1, 4 and 5 are partners in the Trilateral Euregio Cluster (TEC). 
First, low triangularity $\left(\delta_{\mathrm{u}}=0.19\right)$ in septum configuration. In this case large $\mathrm{D}_{2}$ fuelling rates lead to confinement degradation towards L-mode. The seeding of Ar during the $\mathrm{D}_{2}$ fuelling phase gives rise to a density close to the Greenwald value. After the switch-off of the $\mathrm{D}_{2}$ gas fuelling ('afterpuff' phase), the confinement recovers to H-mode quality whereas the density stays near the value reached at the end of the main fuelling phase and $Z_{\text {eff }}$ stays close to or below 2. Acting on the refuelling of $A r$ and $D_{2}$ in the 'afterpuff' phase allows us to improve the stationarity of the high performance phase while maintaining up to the end of the heating phase the good confinement, density and radiation level.

Second, high triangularity $\left(\delta_{\mathrm{u}}=0.45\right)$ in vertical target configuration. In this case large fuelling rates do not lead to strong confinement degradation and the $\mathrm{D}_{2}$ fuelling is applied continuously throughout the discharge. A radiated power fraction of up to $70 \%, \mathrm{H} 98(\mathrm{y}, 2)=0.9$ at $\beta_{\mathrm{n}}=2.1$ and $n=1.15 n_{\mathrm{GW}}$ - together with the formation of a radiating mantle and moderate $Z_{\text {eff }}$-are achieved in this scenario. Furthermore, there are indications of significantly reduced heat load on the divertor target plates.

\section{Introduction}

The ITER reference operational scenario for pulsed operation at $Q=10$ is the ELMy H-mode with a confinement characterized by $\mathrm{H} 98(\mathrm{y}, 2)=1$ at $f_{\mathrm{GW}}=n / n_{\mathrm{GW}}=0.85$ and with $\beta_{\mathrm{n}}=1.8$, where $\mathrm{H} 98(\mathrm{y}, 2)$ is the enhancement factor over the IPB98(y, 2) scaling law [1] and $f_{\mathrm{GW}}$ is the Greenwald factor, i.e. the density normalized to the empirical Greenwald density limit $n_{\mathrm{GW}}=I_{\mathrm{p}} / \pi a^{2}\left(10^{20} \mathrm{~m}^{-3}, \mathrm{MA}, \mathrm{m}\right)$ [2]. Previous experiments on JET and other tokamaks [3] have shown that the energy confinement degrades when raising the density towards $n_{\mathrm{GW}}$ and that the maximum achievable density for a given confinement is increasing with the triangularity $\delta$ [4]. Hence, the necessity to develop scenarios combining high confinement and $\beta_{\mathrm{n}}$ at high density. However, high confinement is generally accompanied by large type I ELMs of low frequency causing severe transient power loads on the divertor target plates and likely to shorten the lifetime of these elements. Radiating a substantial part of the energy over greater surface areas may reduce the power load. This can be achieved by the seeding of low $Z$ impurities into the plasma, trying to create a radiating mantle. Impurity seeding can also lead to confinement improvement mainly at high density. This has been shown in the pioneering work on TEXTOR (RI-mode) in a limiter machine [5] and afterwards on several divertor tokamaks: ASDEX-U [6], DIII-D [7] and recently on JT60-U [8]. In the H-mode the benefit of impurity seeding is to extend high confinement to higher densities and first experiments on JET have shown this favourable trend [9].

The present experiments aim at defining ELMy H-mode integrated scenarios fulfilling at the same time the main requirements for ITER with the help of impurity (here Ar) seeding without altering significantly the purity of the plasma centre. Different plasma configurations, main gas puffing $\left(\mathrm{D}_{2}\right)$ and impurity gas $(\mathrm{Ar})$ seeding schemes were explored. We have shown that discharges meeting all the normalized parameters needed for ITER could be achieved in steady state and in a reproducible way in two classes of discharges: (i) low triangularity discharges with the X-point lying on the top of the septum and (ii) high triangularity discharges with a plasma shape very close to the one proposed for ITER. The obtained performance depends on the plasma configuration, the details of the deuterium and impurity gas fuelling scheme used and on the applied additional heating power. In what follows we give a systematic 
account of the subtle interplay of these parameters on the discharge characteristics. A first overview of these results has been presented in $[10,11]$.

\section{2. 'Septum' experiments}

A first series of experiments was carried out in the 'septum' configuration, i.e. with the X-point lying on the dome of the MkIIGB divertor (figure 1, two left figures). This configuration was chosen because this diverted plasma configuration is limited toroidally by the septum and is therefore closest to the configuration limited by the toroidal pumped belt limiter of TEXTOR where the promising RI-mode, combining many attractive features, was developed [5]. However, the JET septum configuration does not behave like a pumped limiter configuration, but, on the contrary, is characterized by a low $\mathrm{L}-\mathrm{H}$ power threshold; therefore, an $\mathrm{H}$-mode is kept even if a substantial fraction of the power is radiated. Operational range explored is: $I_{\mathrm{p}}=2.5 \mathrm{MA}, B_{\mathrm{t}}=2.4 \mathrm{~T}, q_{95} \approx 3.05, P_{\mathrm{NBI}}=11-15 \mathrm{MW}, P_{\mathrm{ICRH}}=0-3 \mathrm{MW}$.

\subsection{Septum low-triangularity discharges $\left(\kappa=1.65, \delta_{1}=0.24, \delta_{\mathrm{u}}=0.18\right)$}

We distinguish two phases in these discharges (figure 2). The first phase is called the 'puff' phase, where large deuterium $\left(\mathrm{D}_{2}\right)$ fuelling rates are used to rise the density. The second phase is the 'afterpuff' phase starting at the end of the large $\mathrm{D}_{2}$ puff. The strong $\mathrm{D}_{2}$ puff in the 'puff' phase causes a confinement degradation. However, confinement recovers in the 'afterpuff' phase, but then at the much higher density reached at the end of the 'puff' phase. A first means to prevent a decay of the density in the 'afterpuff' phase is to apply much smaller $\mathrm{D}_{2}$ puffs in the 'afterpuff' to refuel without significant confinement degradation, but this is
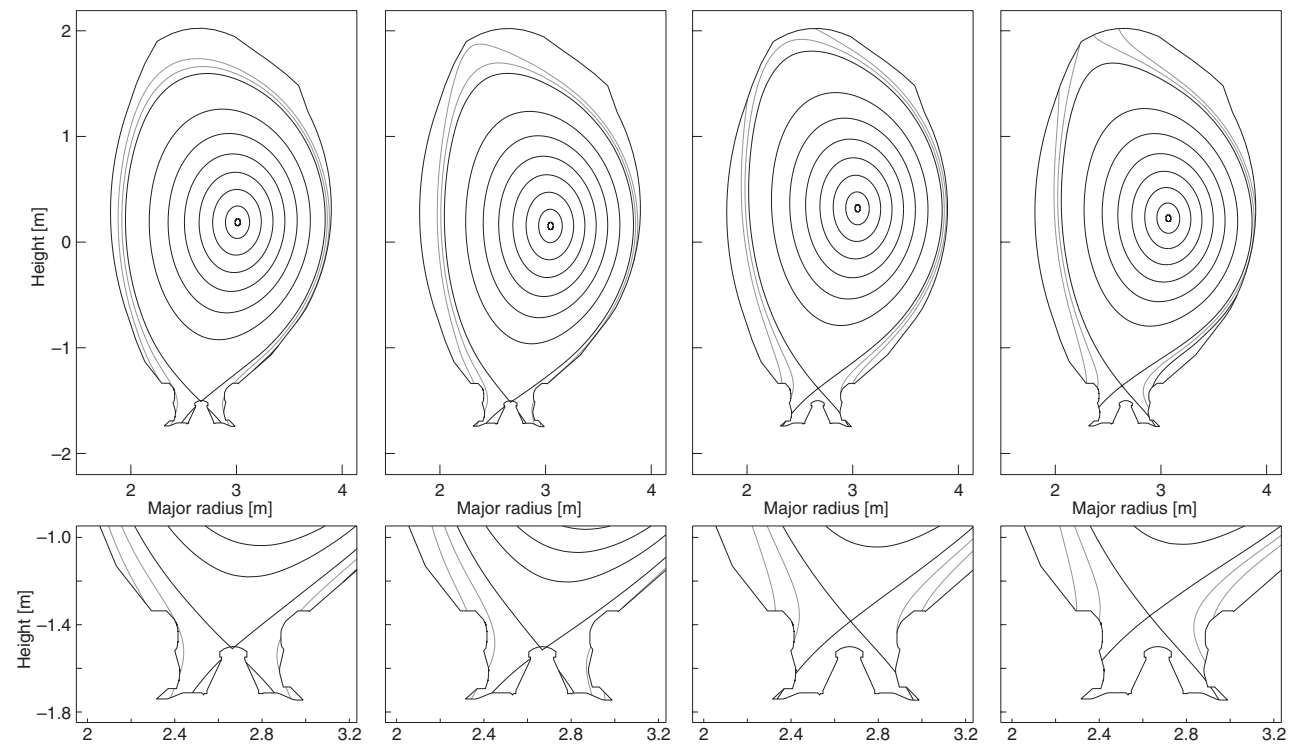

Figure 1. Different studied configurations with zoom on the X-point region in the bottom figures. From left to right: septum (i.e. with the X-point lying on the dome of the MkIIGB divertor) low triangularity, septum high triangularity, vertical target (i.e. with a lower single null above the dome of the divertor and the strike points on the vertical target plates of the divertor) medium triangularity and vertical target EHT. 


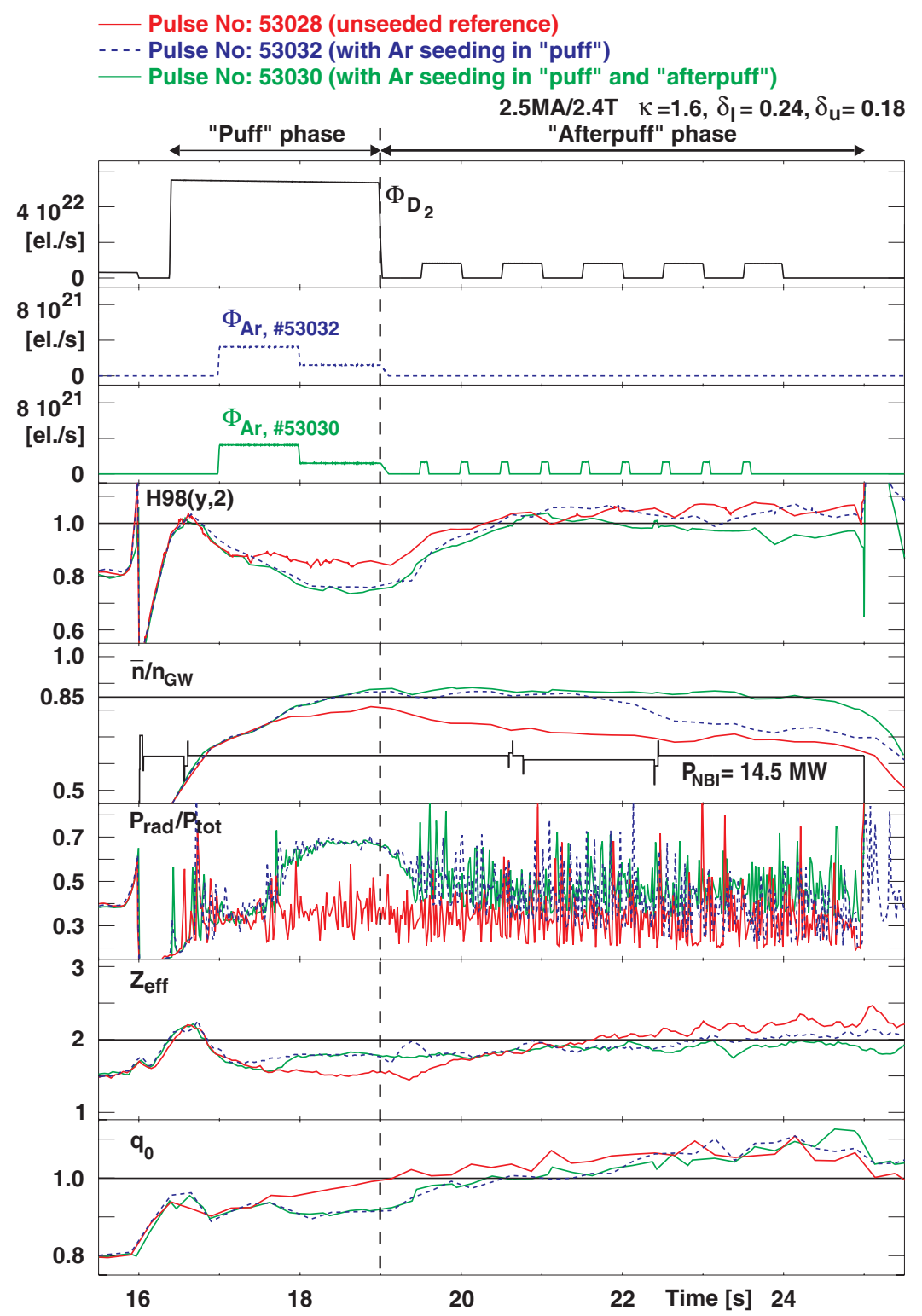

Figure 2. Time traces of global plasma parameters for three discharges in septum low triangularity configuration: unseeded reference (plain red), with impurity seeding in the 'puff' phase only (dashed blue) and an optimized discharge with impurity seeding both in the 'puff' and in the 'afterpuff' phases (plain green): gas fuelling rates (in electrons $\mathrm{s}^{-1}$, assuming fully stripped ions) of $\mathrm{D}_{2}$ (the same for all three displayed discharges) and of Ar for the two impurity seeded discharges, confinement enhancement factor H98(y, 2), Greenwald fraction, neutral beam power, total radiated power fraction, $Z_{\text {eff }}$ and central safety factor $q_{0}$.

generally insufficient. Although the primary reason for impurity seeding is to establish a radiating mantle in order to reduce peak heat loads on first wall components during ELMs, impurity seeding is also an additional means to keep the high density, as it increases the product of fuelling efficiency and particle confinement time, as explained below. Different impurity 


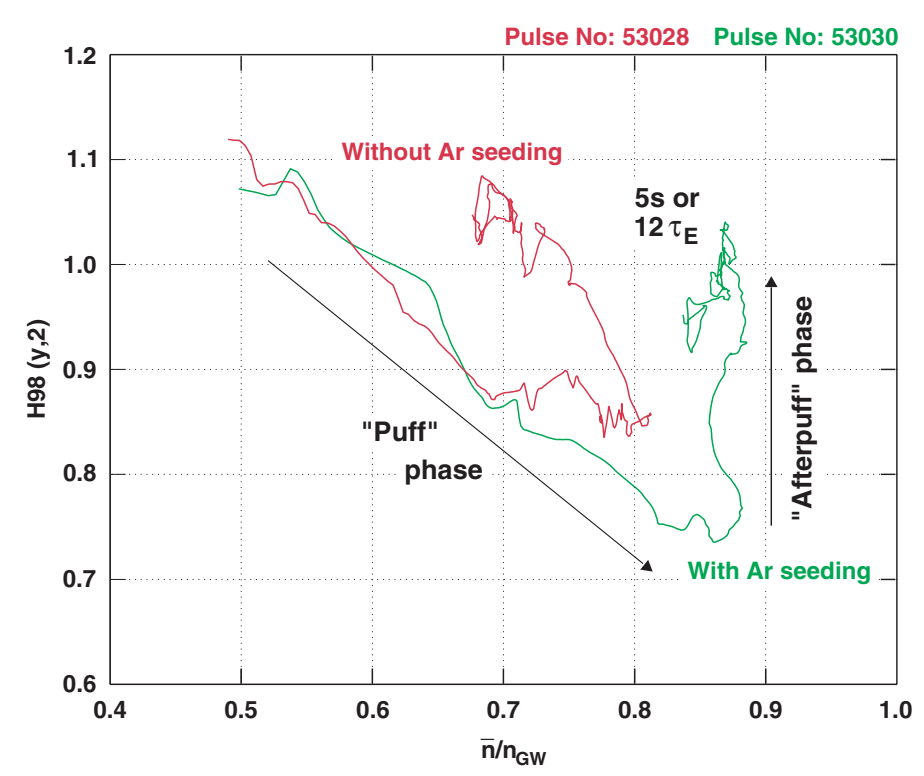

Figure 3. Trajectories of the unseeded reference and the optimized impurity seeded discharge of figure 2 in the space $\left(n / n_{\mathrm{GW}}, \mathrm{H} 98(\mathrm{y}, 2)\right)$ showing the higher density and performance reached by the Ar seeded discharge. The high performance is sustained for about $5 \mathrm{~s}$, i.e. 12 energy confinement times.

seeding schemes are illustrated in figure 2, where a reference discharge (red traces) without impurity seeding is compared with two discharges with different Ar impurity seeding schemes: dashed blue traces correspond to a discharge with Ar seeding in the 'puff' phase only (Ar fuelling rates in figure 2, box 2) and green traces correspond to an optimized discharge with Ar seeding during both the 'puff' and 'afterpuff' phase (Ar fuelling rates in figure 2, box 3 ). The $\mathrm{D}_{2}$ puffing scheme is shown in figure 2, box 1 and is common to all three displayed discharges.

With Ar seeding in the 'puff' phase only, the density in this phase rises to values even closer to the Greenwald value (figure 2, box 5) but at the expense of a further confinement degradation (figure 2, box 4). There is, however, still a density decay in the 'afterpuff'. Small amounts of Ar during the 'afterpuff' cure this problem and the result is a discharge with simultaneously normalized density, confinement and $\beta$ values $\left(\beta_{\mathrm{n}}=1.8\right)$ as required for ITER, maintained during 12 energy confinement times or $5 \mathrm{~s}$, (for this discharge, the duration is limited by the maximum duration of the additional heating power). The effect of Ar seeding on the performances is summarized in figure 3 showing the evolution of the unseeded reference discharge and of the optimized Ar seeded discharge in the space $\left(n / n_{\mathrm{GW}}, \mathrm{H} 98(\mathrm{y}, 2)\right)$ : higher densities are reached with the seeding of Ar in the 'puff' phase and confinement is recovered in the 'afterpuff' phase, keeping the density reached at the end of the 'puff' phase.

It is worthwhile to note that the value of $Z_{\text {eff }}$ measured by visible bremsstrahlung is maintained constant around 2 and is even lower in the seeded discharges than in the unseeded reference discharge due to the higher density reached (figure 2, box 7). Charge exchange measurements show a low central concentration of Ar in the centre of the discharge $\left(C_{\mathrm{AR}} \approx 0.05 \%\right)$ (figure 4). This confirms the low contribution of the seeded impurity to the $Z_{\text {eff }}$. Nevertheless, in this type of discharge a continuous increase of $q_{0}$ is observed (figure 2, box 8). This leads, when $q_{0}>1$, to the loss of sawtooth activity and a start of impurity accumulation. The impurity accumulation when using higher Ar levels was avoided and hence the stationarity of the discharges improved by maintaining the sawtooth activity with central ICRF heating in order 


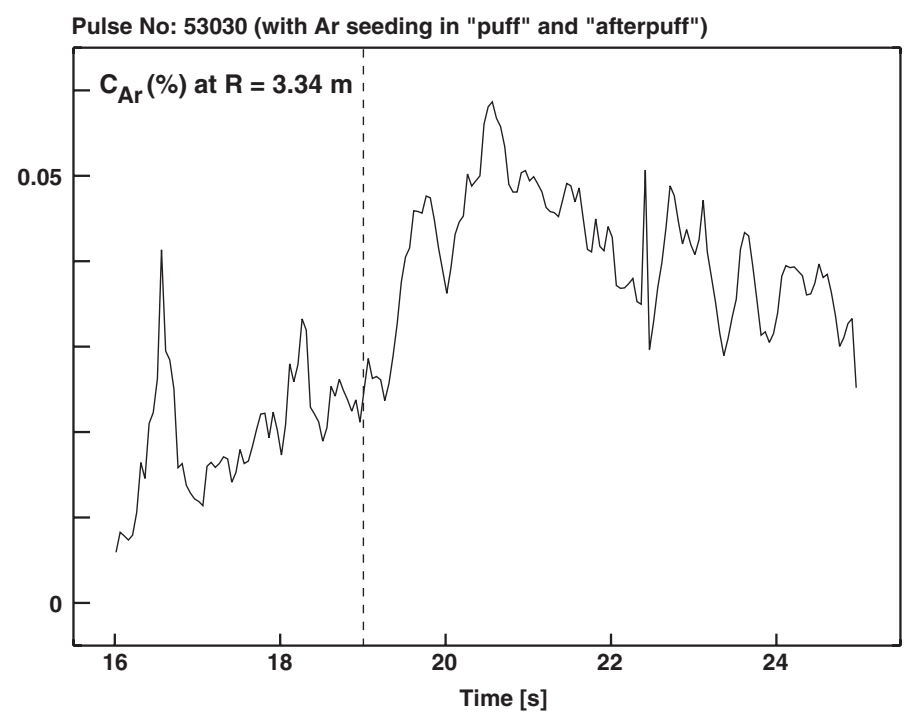

Figure 4. Ar concentration at $R=3.34 \mathrm{~m}$ (measured by charge exchange spectroscopy) for the optimized Ar seeded septum low triangularity discharge of figure 2, The dashed line represents the start of the 'afterpuff' phase.

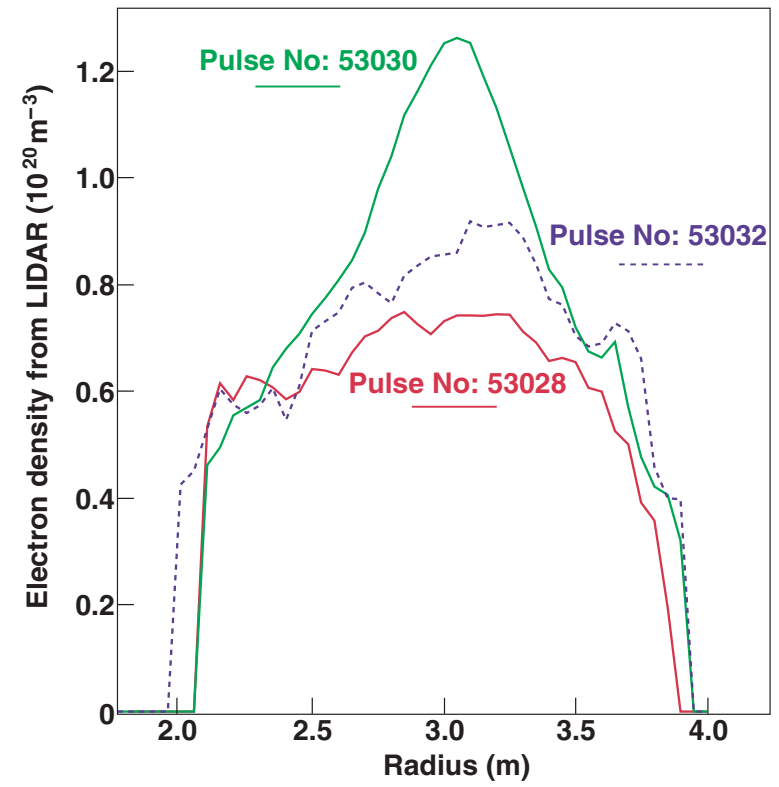

Figure 5. Density peaking for the three shots of figure 2 taken at 22.9 s. Pulse 53028: no impurity seeding; pulse 53032: Ar seeding in 'puff' only; pulse 53030 with Ar seeding in both 'puff' and 'afterpuff'.

to keep $q_{0}<1$. The steady rise of $q_{0}$ observed is indeed slowed down when ICRH is added in these discharges $[13,14]$.

Another favourable feature is the density peaking (figure 5) without rise in $Z_{\text {eff }}$. This indeed allows a certain margin in the confinement requirement in the case of a reactor. The contribution 
of the seeded impurity to central density is estimated from the measurement of the central Ar concentration to be less than $1 \%$. The radiated power fraction in the 'afterpuff' rises from $35 \%$ for the unseeded case up to $45 \%$ in the seeded case, the radiation increase coming mainly from inside the separatrix in the septum region. The radiation profiles obtained by the Abel inversion of the bolometric data show the formation of a radiating belt but accompanied by a non-negligible contribution of the central radiation. This contribution can be lowered by application of central heating which decreases the central Ar concentration and, in case of sufficiently high central temperature, increases its degree of ionization [14].

The ELM behaviour is modified with Ar seeding. In the 'puff' phase, there is a transition from types I to III ELMs, consistent with a further confinement degradation in this phase. In the 'afterpuff' phase, type I ELMs are recovered, again consistent with the recovery of the $\mathrm{H}$-mode confinement quality. This is accompanied by a reduction of the power flux through the separatrix and hence a reduced ELM frequency. Also, the energy loss per ELM $\left(\Delta W_{\text {dia }} / W_{\text {dia }}\right)$ is lowered by $35 \%$ as measured by the fast diamagnetic measurement [15]. In the seeded as well as in the unseeded cases, the ELM energy loss normalized to the pedestal energy $\left(\Delta W_{\mathrm{ELM}} / W_{\text {ped }}\right)$ is well correlated with the collisionality of the pedestal plasma, indicating a clear link between the pedestal plasma parameters and the ELM characteristics (energy loss and frequency) [17].

In stationary conditions, the product of the fuelling efficiency and the particle confinement time is roughly equal to the total number of particles in the plasma divided by the recycling flux: $f \tau_{\mathrm{p}} \approx N_{\text {tot }} / \Phi_{\text {recycling, }}$, where the fuelling efficiency $f$ is the probability for a neutral atom starting at a material surface to reach the confined plasma [18]. In the 'puff' as well as in the 'afterpuff' phase, the recycling fluxes (measured at the wall and at the X-point) are not affected by the seeding of Ar while the density is higher. Keeping the same density, the recycling fluxes drop when cutting the large D puff. Consequently, $f \tau_{\mathrm{p}}$ is higher in the Ar seeded cases and increases when going from 'puff' to 'afterpuff'.

Septum discharges are sensitive to the levels of $\mathrm{D}_{2}$ puffing and Ar seeding in the 'afterpuff'. Indeed, higher levels of Ar, besides a higher total radiated power fraction, lead to the observation of the following four effects: (i) as for lower Ar levels continuous increase of the central $q$ is seen (flattening of the $q$ profile or even appearance of central shear reversal) leading to the disappearance of the sawteeth when $q_{0}>1$ followed by central impurity accumulation, (ii) enhanced MHD activity [19], (iii) confinement loss and (iv) appearance of ELM-free phases. The causality between these effects needs further study but the current understanding is that the changes in $q_{0}$ inducing sawteeth suppression trigger the enhanced MHD activity and hence the loss of confinement [13].

\subsection{Septum high triangularity discharges $\left(\kappa=1.73, \delta_{1}=0.29, \delta_{\mathrm{u}}=0.33\right)$}

Discharges with the X-point on the septum at a higher triangularity (figure 1, second figure from the left) showed qualitatively the same effects as described above. However, somewhat higher densities are reached at the end of the 'puff' phase without Ar seeding. In contrast to the septum low triangularity discharges, seeding of Ar during the 'puff' phase does not cause a further increase in the density achieved. A scan of the distance between the plasma and the wall did not reveal a clear influence of the proximity of the walls. The better fuelling efficiency is, therefore, attributed to the higher $\delta$ of the plasma. Ar seeding during the 'afterpuff' phase results in a $10 \%$ higher radiation power fraction but no noticeable effect is seen on the density behaviour. $Z_{\text {eff }}$ in the 'afterpuff' remains around 2 and $\beta_{\mathrm{n}}$ around 1.8. 


\section{Vertical target configuration}

\subsection{Medium triangularity $\left(\kappa=1.73, \delta_{1}=0.27, \delta_{\mathrm{u}}=0.35\right)$}

Discharges with the strike points on the vertical target plates of the divertor at medium triangularity (figure 1, third figure from the left) show a behaviour similar to the septum high triangularity case. Operational parameters were: $I_{\mathrm{p}}=2.5 \mathrm{MA}, B_{\mathrm{t}}=2.4 \mathrm{~T}, q_{95} \approx 3.1$, $P_{\mathrm{NBI}}=12.5 \mathrm{MW}, P_{\mathrm{ICRH}}=0 \mathrm{MW}$. The 'puff'-'afterpuff' scenario is also used for these discharges. In the 'puff' phase, the large $\mathrm{D}_{2}$ fuelling rates used to rise the density deteriorate the confinement, the degradation in the 'puff' phase being stronger in the Ar seeded discharges than in the reference discharges without Ar seeding. Nevertheless, in all discharges good $\mathrm{H}$-mode confinement is recovered in the 'afterpuff' phase. The density obtained at the end of the 'puff' phase is not higher in the impurity seeded discharges than in the reference discharges without Ar seeding and the density stays rather low as compared to the septum high and low triangularity cases $\left(n=0.75-0.8 n_{\mathrm{GW}}\right)$ (figure 6$)$. For the discharges discussed in this paper, an increase is observed in the radiated power fraction from $40 \%$ to $60 \%$ with Ar seeding in the 'afterpuff' phase, with a slightly higher value for $Z_{\text {eff }}$ (from 1.8 to 2 ) at constant $\beta_{\mathrm{n}} \approx 1.8$. The ELM frequency is clearly smaller in the 'afterpuff' with impurity seeding, the energy loss

Pulse No: 52161 (with Ar seeding in "puff" and "afterpuff") 2.5MA/2.4T $\kappa=1.73, \delta_{1}=0.27, \delta_{u}=0.35$

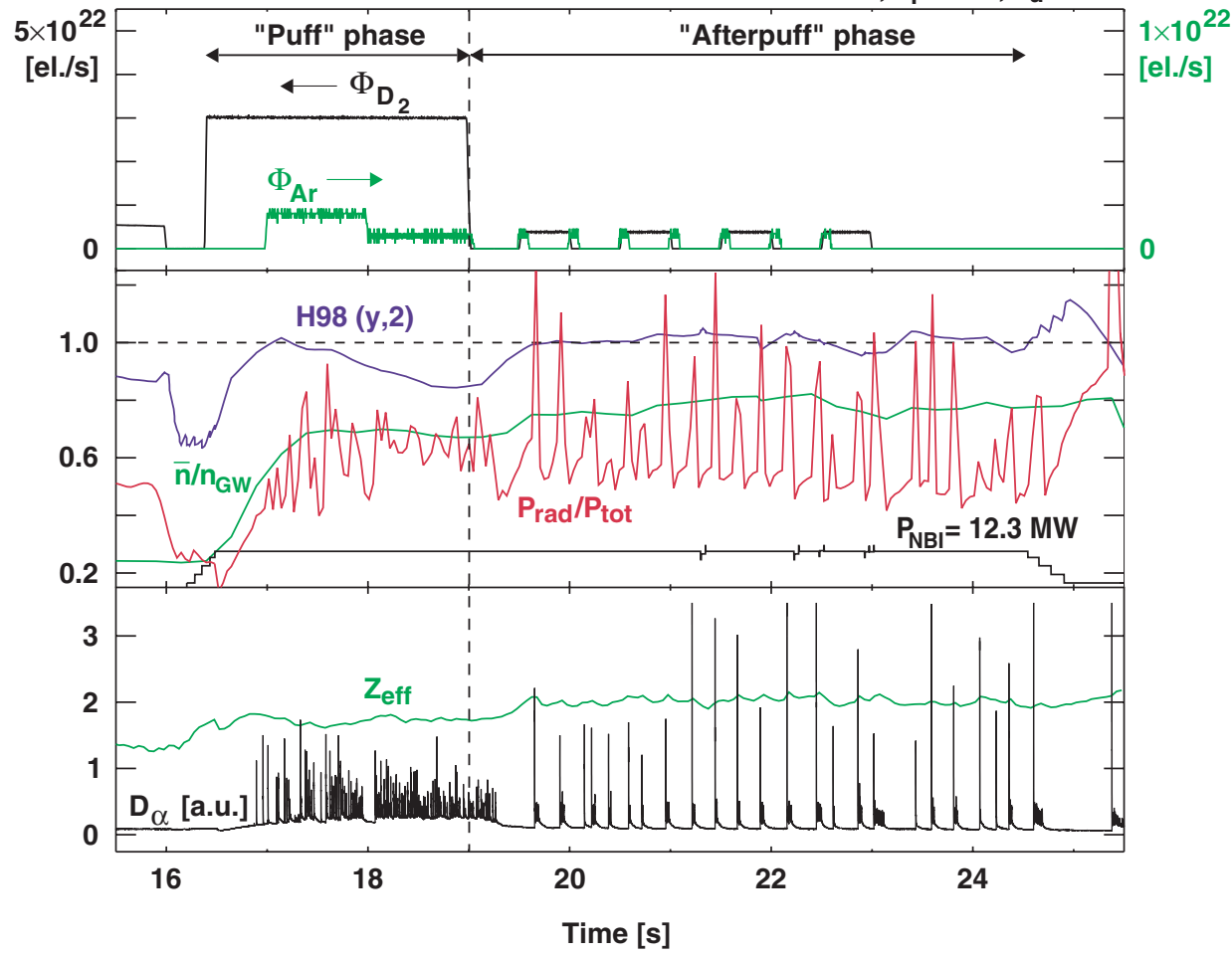

Figure 6. Example of an Ar seeded vertical target medium triangularity discharge using the 'puff'-'afterpuff' scheme. Top box: time traces of the fuelling rates for $\mathrm{D}_{2}$ and $\mathrm{Ar}$ (in electrons s ${ }^{-1}$ assuming fully stripped ions). Middle box: time traces of the confinement enhancement factor $(\mathrm{H} 98(\mathrm{y}, 2))$, the Greenwald fraction $\left(n / n_{\mathrm{GW}}\right)$, the total radiated power $\left(P_{\mathrm{rad}} / P_{\mathrm{tot}}\right)$ and the neutral beam power. Bottom box: time traces of the $D_{\alpha}$ brilliance and of $Z_{\text {eff }}$. 
per ELM ( $\left.\Delta W_{\text {dia }} / W_{\text {dia }}\right)$ being estimated to be $30 \%$ lower in the impurity seeded discharges as compared to the reference discharges without Ar seeding [15].

\subsection{Extremely high triangularity $\left(\kappa=1.7, \delta_{1}=0.35, \delta_{\mathrm{u}}=0.45\right)$}

Previous experiments on JET and other tokamaks [3] have shown that not only the energy confinement degrades when raising density towards $n_{\mathrm{GW}}$ but also that this degradation occurs at a higher density when the triangularity is increased: in this case, large continuous $\mathrm{D}_{2}$ fuelling rates may not be too detrimental for the confinement. The 'puff'-'afterpuff' scheme is consequently not needed for this scenario. Small amounts of Ar were seeded in such high $\delta$ discharges $\left(\delta_{\mathrm{u}} \approx 0.45\right)$ with large heating power (with respect to the $\mathrm{L}-\mathrm{H}$ power threshold) and continuous large $\mathrm{D}_{2}$ fuelling. The $\mathrm{X}$-point here is well above the septum and the strike points laying at different heights on the vertical target plates (figure 1, right figure). These plasmas have been realized at $I_{\mathrm{p}}=2.3 \mathrm{MA}, B_{\mathrm{t}}=2.4 \mathrm{~T}, q_{95} \approx 3.05, P_{\mathrm{NBI}} \approx 14 \mathrm{MW}$, $P_{\text {ICRH }} \approx 2 \mathrm{MW}$ and typical plasma parameters obtained are displayed in figure $7:$ normalized

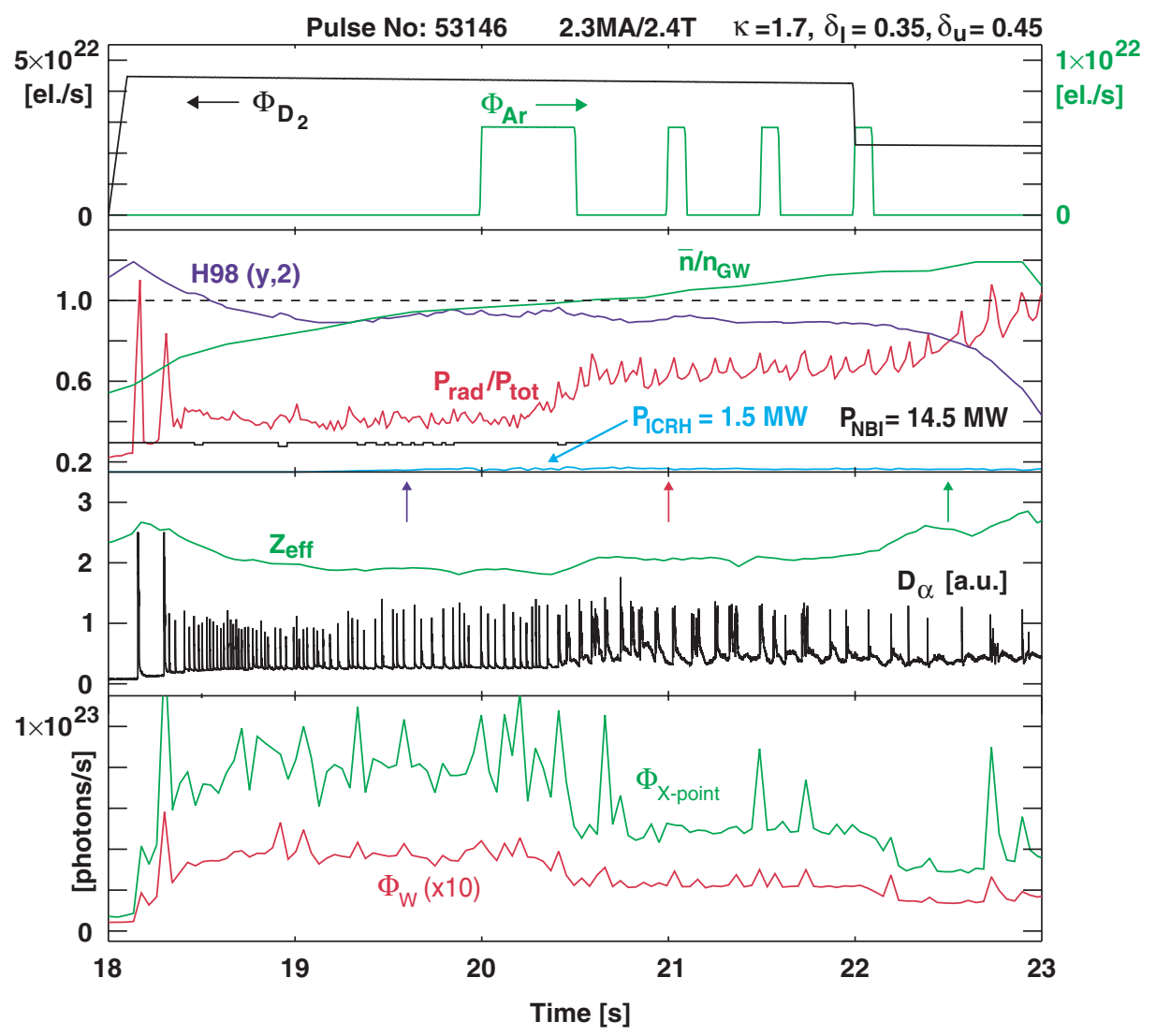

Figure 7. Time traces of global plasma parameters for an Ar seeded discharge in the EHT configuration. Top box: $\mathrm{D}_{2}$ and Ar fuelling rates (in electrons $\mathrm{s}^{-1}$, assuming fully stripped ions). Second box: confinement enhancement factor $(\mathrm{H} 98(\mathrm{y}, 2))$, Greenwald fraction $\left(n / n_{\mathrm{GW}}\right)$, total radiated power $\left(P_{\text {rad }} / P_{\text {tot }}\right)$, neutral beam and ICRH powers. Third box: $\mathrm{D}_{\alpha}$ brilliance and $Z_{\text {eff }}$. Bottom box: total $\mathrm{D}_{\alpha}$ photon fluxes at the X-point and at the wall. Density profiles for the times indicated by the arrows in box 3 are shown in figure 9 . 
density $f_{\mathrm{GW}} \approx 1$ as well as $\mathrm{H} 98(\mathrm{y}, 2)=0.9-1$ [20]. In this case, the seeding of Ar (starting at $20 \mathrm{~s}$ in figure 7) enhances the particle confinement time and leads to a continuous rise of the density to even higher values. $Z_{\text {eff }}$ is slightly higher $\left(\Delta Z_{\text {eff }}=0.2\right)$ and there is a slight confinement penalty $(\Delta \mathrm{H} 98(\mathrm{y}, 2) \leqslant 5 \%)$ with $\mathrm{H} 98(\mathrm{y}, 2)$ nevertheless remaining close to unity. The contribution of Ar to the increase of the total plasma density, as deduced from the Ar concentration profile (from charge exchange measurements), is less than $2 \%$ and the total electron density profile remains flat (figure 9, top box). Peak values simultaneously obtained in these discharges are $\mathrm{H} 98(\mathrm{y}, 2)=0.9, \beta_{\mathrm{n}}=2.1$ and $n=1.15 n_{\mathrm{GW}}$ at a radiated power fraction of up to $70 \%$.

Both the $\mathrm{D}_{2}$ gas fuelling rate and the Ar seeding rate influence the product $f \tau_{\mathrm{p}}$ ( $f$ being the fuelling efficiency as defined earlier and $\tau_{\mathrm{p}}$ the particle confinement time). This is shown in figure 8: for a given total radiated power fraction $f \tau_{\mathrm{p}}$ decreases with the increase of the $\mathrm{D}_{2}$ fuelling rate, and for a given $\mathrm{D}_{2}$ fuelling rate $f \tau_{\mathrm{p}}$ increases with the increase of the total radiated power fraction (which depends on the Ar fuelling rate). Consequently the density depends on these fuelling rates. In figure 7, the seeding of Ar at $20 \mathrm{~s}$ leads to an increase of the product $f \tau_{\mathrm{p}}$ as indicated by the increase of the density and the decrease of the $\mathrm{D}_{\alpha}$ fluxes at the wall and at the X-point. A similar effect is observed at $22 \mathrm{~s}$ when the $\mathrm{D}_{2}$ fuelling rate is reduced.

The large density obtained after $22 \mathrm{~s}$ results in a less centrally deposited beam power (figure 9). This leads to a lower central electron temperature leading, in turn, to an enhanced central radiation lowering further the central electron temperature. A small amount of ICRH was added to compensate the lack of central heating, but was insufficient to avoid the collapse of the central electron temperature. Power coupling difficulties in the presence of ELMs prevented a further increase of the ICRH power.

The major difference brought by the seeding of Ar resides in the radiation pattern. The total radiated power fraction is higher (going from $45 \%$ to $65 \%$ ) and the increase in radiation comes mainly from the edge of the plasma, creating a radiating mantle. The ratio of bulk radiation to total radiation is displayed in figure $10(a)$ and is higher in the seeded case showing that the radiation is not coming from the divertor region but instead from the bulk of the plasma. The Abel inverted radiation profiles are displayed in figure $10(b)$ and clearly show that the
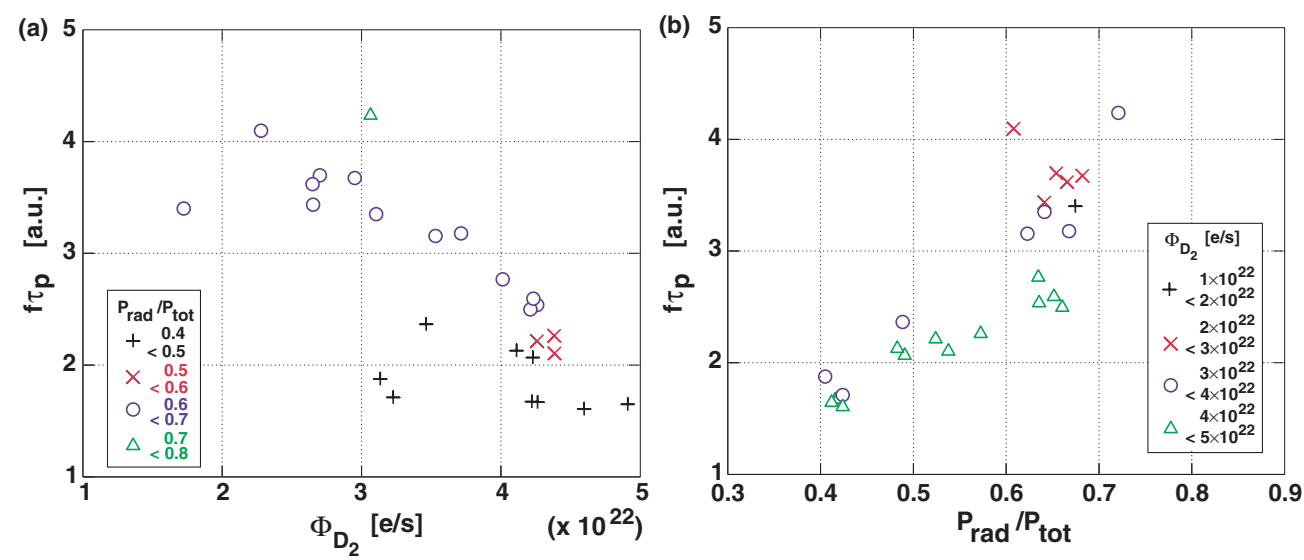

Figure 8. Product of fuelling efficiency and particle confinement time $\left(f \tau_{\mathrm{p}}\right)$ as a function of $(a)$ the $\mathrm{D}_{2}$ fuelling rate with the total radiated power fraction as parameter and as a function of $(b)$ the total radiated power fraction with the $\mathrm{D}_{2}$ fuelling rate as parameter. Data shown are from Ar seeded discharges in the EHT configuration. 

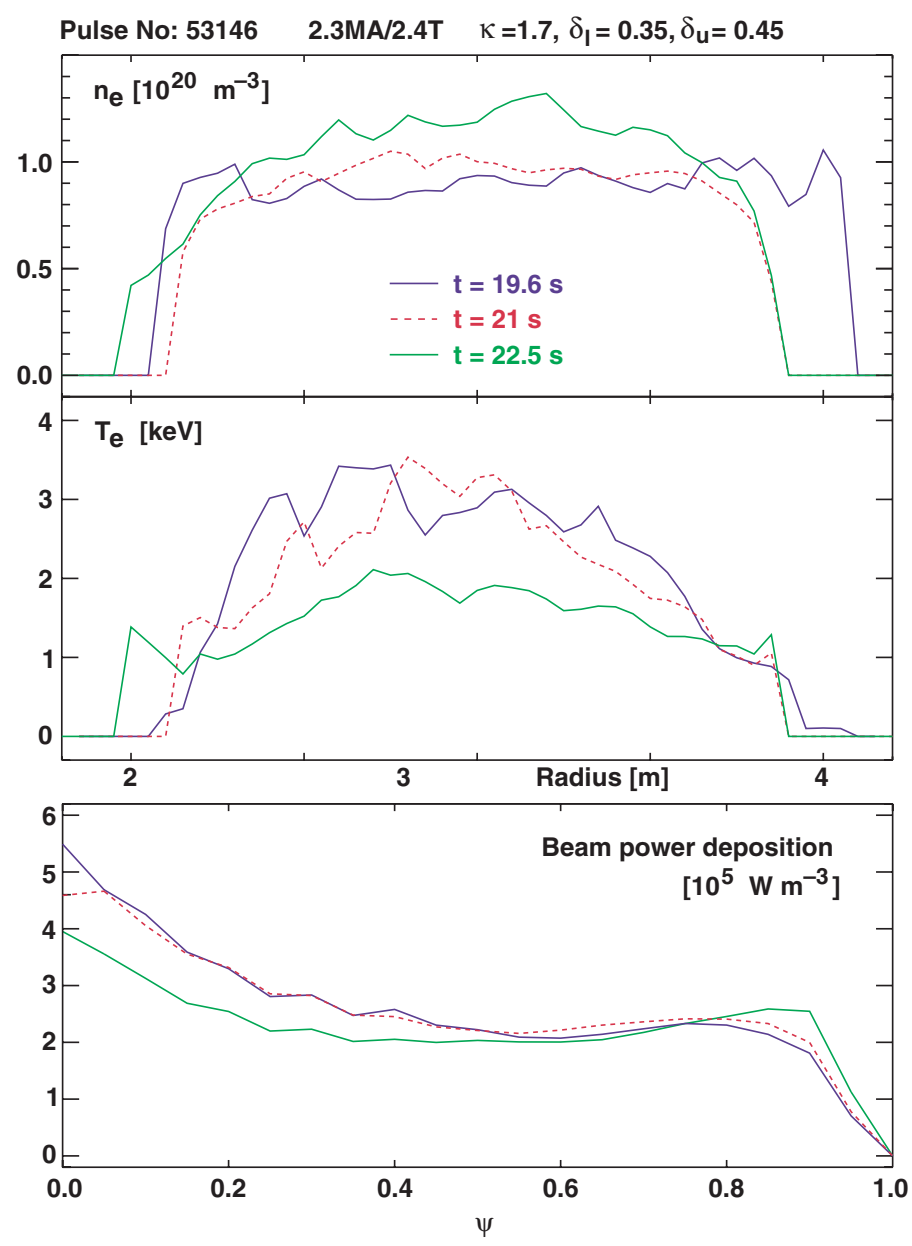

Figure 9. Electron density profiles, electron temperature profiles and beam power deposition profiles for the impurity seeded EHT discharge \#53146 of figure 7.

bulk radiation is mainly localized near the edge of the plasma. The enhanced edge radiation is interpreted as a reduction of the average degree of ionization of the Ar at the edge due to charge exchange processes with the puffed $\mathrm{D}_{2}$ and the slightly lower edge temperature [14]. Charge exchange measurements also show hollow or flat Ar density profiles. As in the case of the septum discharges, the unseeded reference scenario shows a continuous rise in $q_{0}$ throughout the shot. The seeding of $\mathrm{Ar}$ as shown in figure 11 causes a slower temporal evolution of the central $q$.

IR camera measurements show a significant reduction of the divertor target temperature in the Ar seeded case. However, these measurements were up to now performed at a fixed position in the inner and outer divertors and the complete temperature profiles are not available [15]. On the other hand, the decrease of the electron temperature at the strike point on the outer target plate when seeding the Ar (figure 12) gives indications of partial plasma detachment. In this case the ELM power is partly radiated before reaching the target plates of the divertor resulting in a decreased heat load on the plates [15]. It is worthwhile to note that this scenario, as well as the septum low triangularity one, corresponds to low Ar pumping and high Ar recycling in the divertor [21]. 

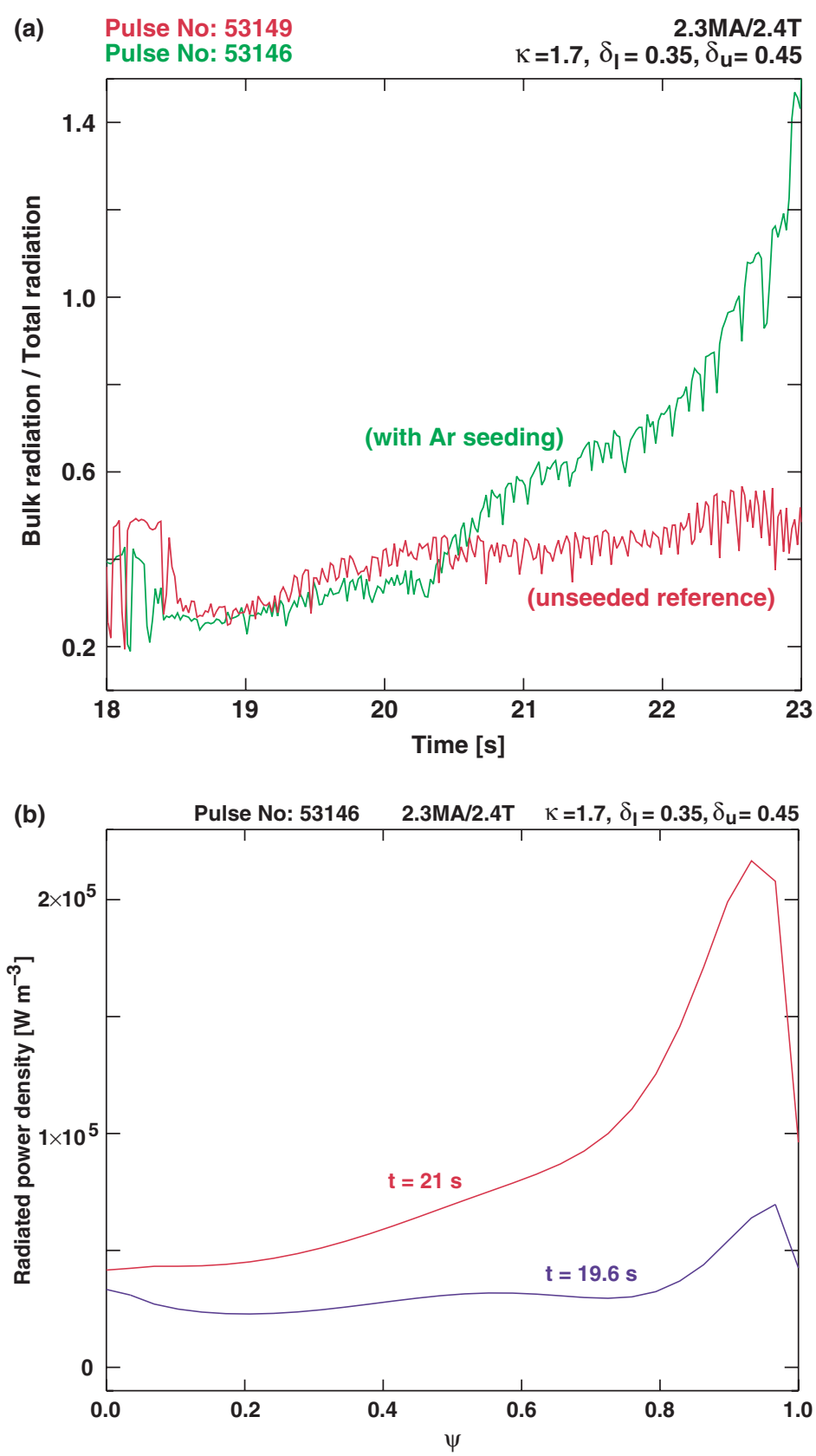

Figure 10. (a) Temporal evolution of the ratio of bulk to total radiation for the Ar seeded EHT discharge of figure 7 and for a reference case without impurity seeding. (b) Abel inverted radiation profiles for the EHT discharge of figure 7 showing the presence of a radiating mantle.

\section{Conclusions}

This work has shown that it is possible to realize simultaneously the normalized values for density $\left(n / n_{\mathrm{GW}} \geqslant 0.85\right)$, confinement $(\mathrm{H} 98(\mathrm{y}, 2) \approx 1)$ and beta $\left(\beta_{\mathrm{n}} \geqslant 1.8\right)$ together with 


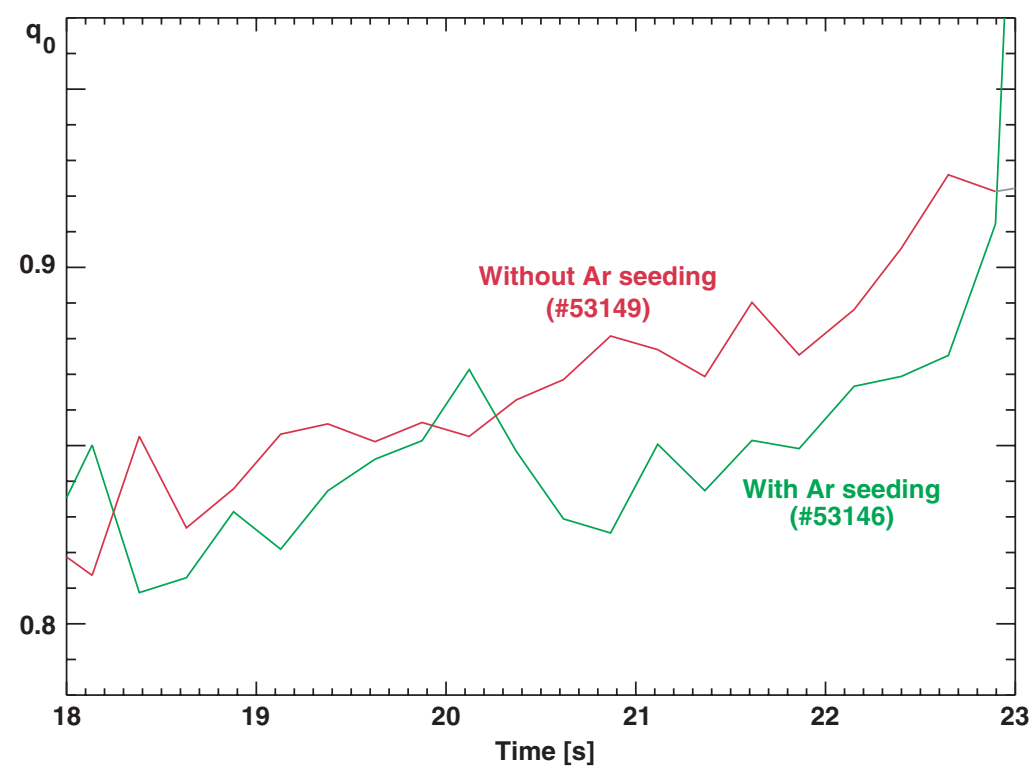

Figure 11. Time evolution of $q_{0}$ for the Ar seeded EHT discharge of figure 7 and for a reference case without impurity seeding.

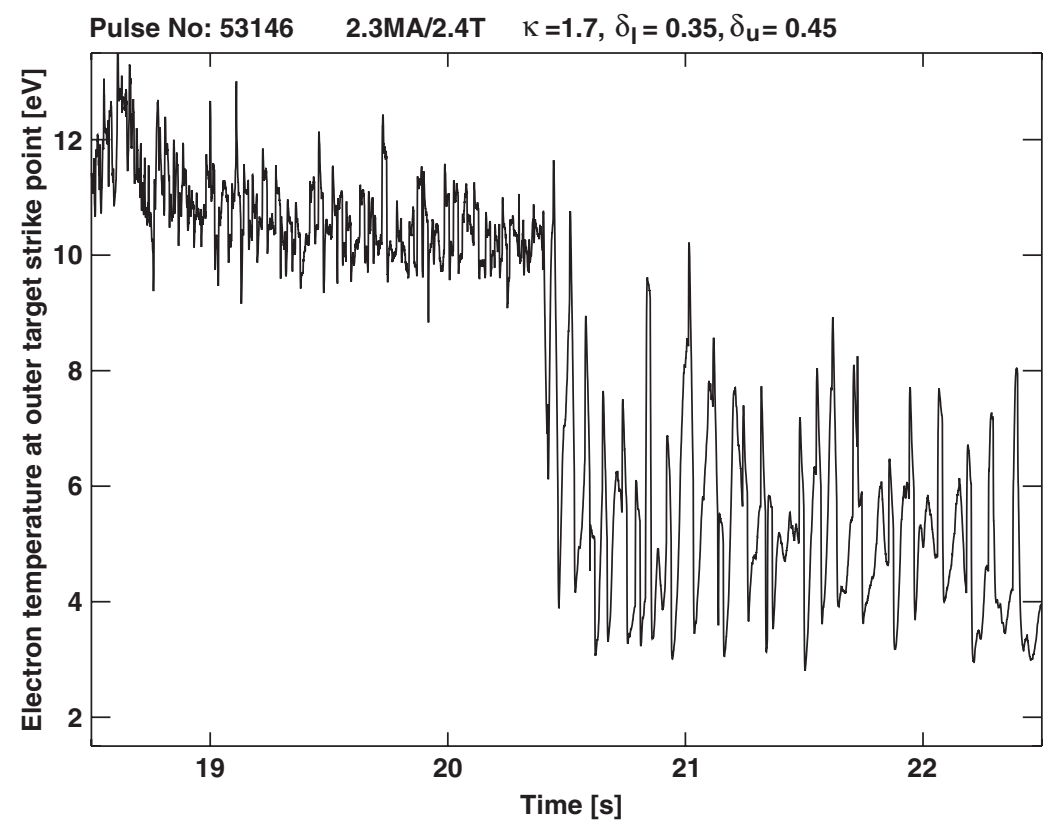

Figure 12. Time evolution of electron temperature at the outer target strike point for the EHT discharge with Ar seeding shown in figure 7.

promising ELM mitigation effects due to the presence of seeded impurities, as required for ITER. Figure 13 summarizes the achieved performance: H98 $(\mathrm{y}, 2)$ and $\beta_{\mathrm{n}} \mathrm{H} 89$ (which is the normalized fusion triple product) as a function of the normalized density $f_{\mathrm{GW}}=n / n_{\mathrm{GW}}$ for the different configurations and gas fuelling schemes explored. The data points shown are 

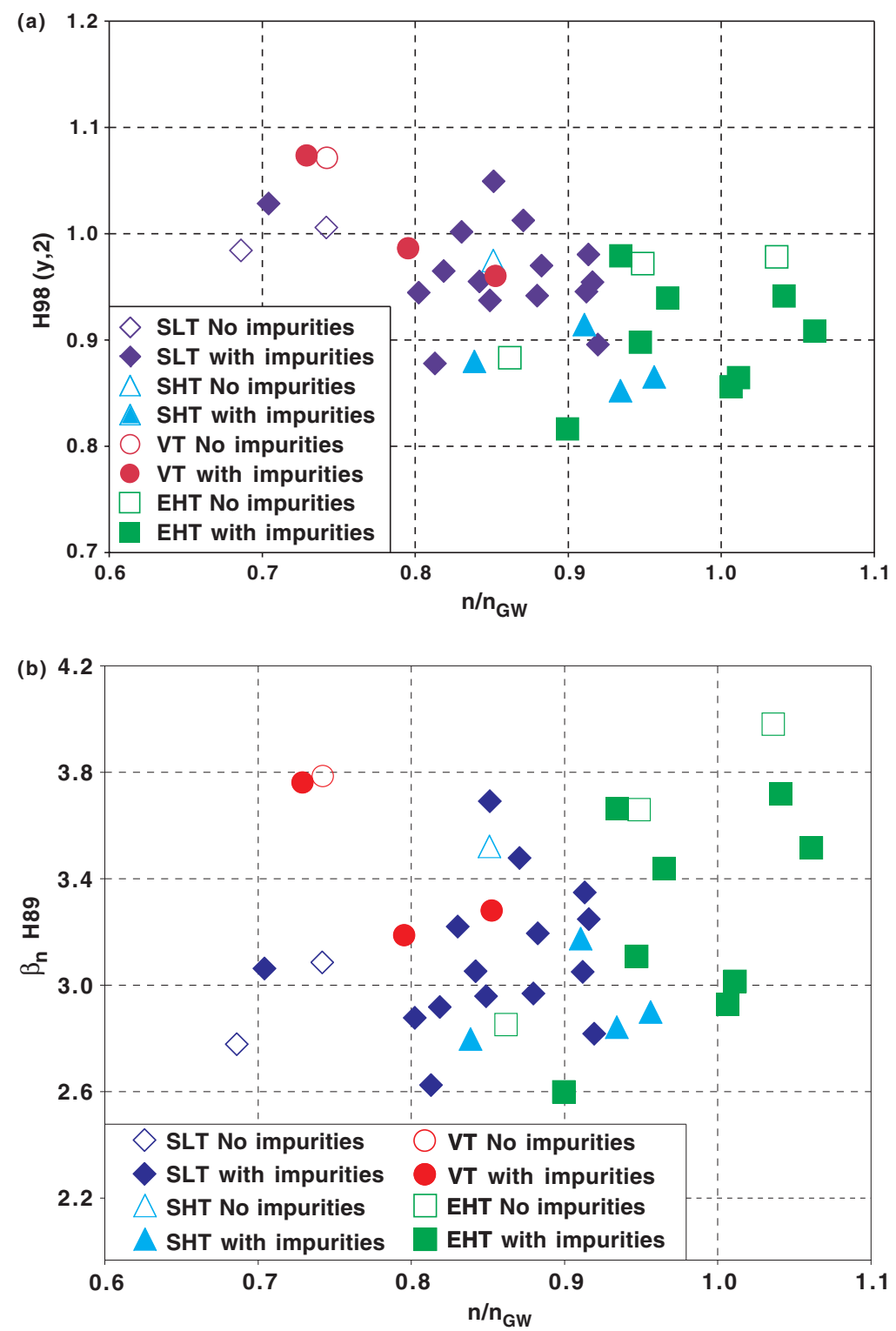

Figure 13. (a) Confinement enhancement factor $(\mathrm{H} 98(\mathrm{y}, 2))$ and $(b)$ normalized fusion triple product $\left(\beta_{\mathrm{n}} \mathrm{H} 89\right)$ vs normalized density $\left(n / n_{\mathrm{GW}}\right)$ for the various configurations described in this paper: septum low $\delta$ (SLT_diamonds), septum high $\delta$ (SHT_triangles), vertical target medium $\delta$ (VT-circles) and vertical target extremely high triangularity (EHT_-squares). Open symbols: unseeded reference discharges; closed symbols: Ar seeded discharges. Averages are taken over stationary intervals of at least three energy confinement times.

averaged over stationary phases of at least three energy confinement times. In the septum low triangularity scenario, the high performance is kept at a higher density in the seeded discharges as compared to the reference discharges without impurity seeding. In the vertical target EHT scenario, the average performances of impurity seeded discharges and reference discharges without impurity seeding are similar and the main benefit of the seeding of the impurity is a significantly higher radiated power fraction. The major results were obtained in two different 
Table 1. Values for several normalized plasma parameters obtained in the septum low triangularity and vertical target EHT discharges with impurity seeding discussed in this paper. $\Delta t / \tau_{\mathrm{E}}$ is the time interval over which the parameters are averaged normalized to the energy confinement time. The values projected for ITER are shown for comparison.

\begin{tabular}{llll}
\hline & $\begin{array}{l}\text { Septum low triangularity } \\
\# 530302.5 \mathrm{MA} / 2.4 \mathrm{~T}\end{array}$ & $\begin{array}{l}\text { Vertical target EHT } \\
\# 531462.3 \mathrm{MA} / 2.4 \mathrm{~T}\end{array}$ & ITER \\
\hline $\mathrm{H} 98(\mathrm{y}, 2)$ & 0.98 & 0.9 & 1.0 \\
$\beta_{\mathrm{n}, \mathrm{th}}$ & 1.75 & 2.0 & 1.81 \\
$n / n_{\mathrm{GW}}$ & 0.86 & $1-1.15$ & 0.85 \\
$Z_{\mathrm{eff}}$ & 1.9 & 2.1 & 1.7 \\
$P_{\mathrm{rad}} / P_{\mathrm{tot}}$ & 0.5 & 0.65 & 0.58 \\
$\kappa, \delta$ & $1.6 / 0.2$ & $1.7 / 0.42$ & $1.84 / 0.5$ \\
$q_{95}$ & 3.0 & 3.1 & 3.0 \\
$\Delta t / \tau_{\mathrm{E}}$ & 12 & 5 & - \\
\hline
\end{tabular}

configurations for which the main non-dimensional parameters are compared to the ITER requirements in table 1.

The first configuration is the 'septum' configuration at low triangularity where the confinement enhancement factor $\mathrm{H} 98(\mathrm{y}, 2)$ could be kept close to unity at high density $\left(f_{\mathrm{GW}} \approx 0.85\right.$ ) for up to 12 energy confinement times. These high densities are reached with seeding of Ar in the 'puff' phase (phase with large $\mathrm{D}_{2}$ fuelling rate in order to raise the density). The confinement drops in this phase due to the sensitivity of the low triangularity discharges to large external $\mathrm{D}_{2}$ fuelling rates. The confinement is recovered in the 'afterpuff' phase (phase starting when the large $\mathrm{D}_{2}$ fuelling is cut) where the density can be maintained by acting on the refuelling of both $\mathrm{D}_{2}$ and Ar. Impurity accumulation can be avoided and stationarity of the discharges can further be improved by applying centrally deposited ICRH in order to keep the sawtooth activity [13]. The total radiated power fraction increases (at least by $10 \%$, depending on the Ar levels) and enhanced edge radiation is observed. Some central radiation is also observed but is reduced by central heating [14]. The ELM frequency is lower with Ar seeding in the 'afterpuff' phase and the ELM losses are reduced [15]. The plasma density profile is peaking (with a minimal contribution to the peaking from the presence of the seeded impurity) and $Z_{\text {eff }}$ remains around 2. Note that on JT60-U, high performances with Ar seeding at $n / n_{\mathrm{GW}}=0.8$ were obtained with a configuration close to the 'septum' one (strike point on the dome top of the divertor) [16].

The second configuration is the so-called extremely high triangularity (EHT) $-\delta_{\mathrm{u}} \approx 0.45$ ) with a continuous large $\mathrm{D}_{2}$ fuelling rate, a small amount of central ICRH and high beam heating power (well above the $\mathrm{L}-\mathrm{H}$ power threshold). Due to the lower sensitivity of the high triangularity discharges to large external $\mathrm{D}_{2}$ fuelling rates, good confinement $(\mathrm{H} 98(\mathrm{y}, 2) \approx 0.9-1)$ is obtained at high density $\left(f_{\mathrm{GW}} \approx 1\right)$. Ar seeding leads to a further increase of the density and a higher radiated power fraction $\left(P_{\text {rad }} / P_{\text {tot }}\right.$ increases from $45 \%$ to $65 \%$ ), the radiation coming mainly from the edge of the plasma. This is obtained at the expense of a slightly higher $Z_{\text {eff }}$ value $\left(\Delta Z_{\text {eff }}=0.2\right)$ and very moderate confinement penalty $(\Delta \mathrm{H} 98(\mathrm{y}, 2) \leqslant 5 \%)$. The density profiles remain flat. There is evidence of reduced heat load on the target plates due to the created radiating mantle and partial detachment of the plasma [15]. It is worthwhile to note that a strong reduction of the ELM heat flux has been observed in similar experiments performed on JT60-U [16]. Peak values reached simultaneously are a radiated power fraction of up to $70 \%, \mathrm{H} 98(\mathrm{y}, 2)=0.9$ at $\beta_{\mathrm{n}}=2.1$ and $n=1.15 n_{\mathrm{GW}}$. Figure 14 shows the evolution of the confinement enhancement factor as a function of the normalized density for different triangularities on JET. Confinement data obtained during stationary phases 


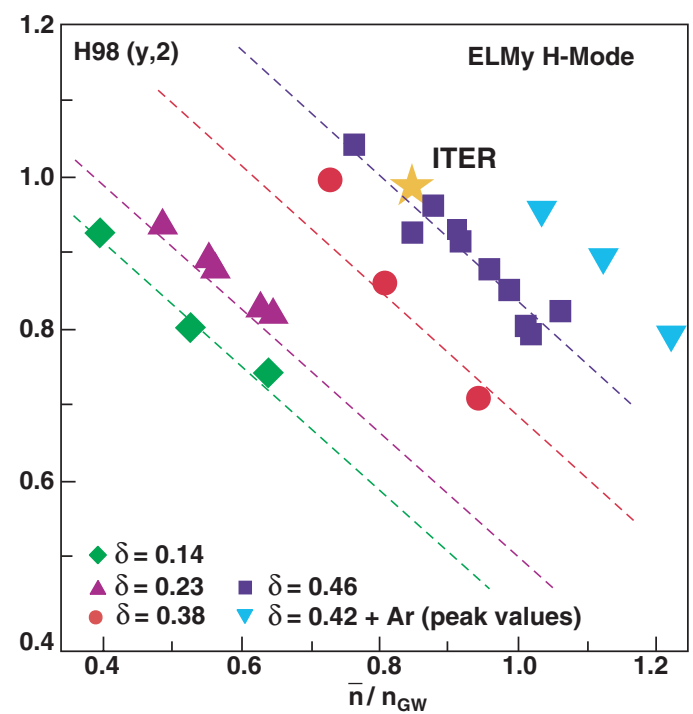

Figure 14. Confinement enhancement factor $H 98(y, 2)$ as a function of the normalized density for different triangularities. Data points from stationary JET database in type I ELM phases. Peak values obtained in the EHT configuration with impurity seeding are indicated by the inverted triangles.

in discharges without impurity seeding follow the dashed curves in this diagram [10]. Data points from stationary phases in unseeded EHT discharges follow the most right dashed curve in this diagram. Data points from peak phases obtained in the EHT configuration with impurity seeding (inverted triangles) are located at even higher density values indicating the potential of this scenario to access high confinement regimes at higher densities.

Further investigation is planned in order to explore and control this higher density regime, to further assess the effect of impurity seeding on ELM mitigation and to improve the control of the density and of the stationarity of the EHT discharge by acting on the fuelling levels, on the strike points position and on the central heating.

\section{Acknowledgment}

This work has been conducted under the European Fusion Development Agreement.

\section{References}

[1] ITER Physics Basis 1999 Nucl. Fusion 392208

[2] Greenwald M et al 1988 Nucl. Fusion 282199

[3] Saibene G et al 1999 Nucl. Fusion 391133

[4] Lomas P et al 2000 Plasma Phys. Control. Fusion 42 B115

[5] Weynants R R et al 1999 Nucl. Fusion 391637

[6] Kallenbach A et al 1996 Plasma Phys. Control. Fusion 382097

[7] Jackson G L et al 2002 Nucl. Fusion 42 28-41

[8] Kubo H et al 2001 Nucl. Fusion 41227

[9] Strachan J D et al 2000 Plasma Phys. Control. Fusion 42 A81-8

[10] Ongena J et al 2001 Plasma Phys. Control. Fusion 43 A11-30

[11] Suttrop W et al 2002 Phys. Plasmas $92103-12$

[12] Ongena J et al 2002 Phys. Plasmas 82188 
[13] Nave M F et al 2001 Proc. 28th EPS Conf. on Controlled Fusion and Plasma Physics (Madeira, 2001) paper P3.009

[14] Puiatti M E et al 2002 Plasma Phys. Control. Fusion 44 1863-78

[15] Jachmich S et al 2002 Plasma Phys. Control. Fusion 44 1879-91

[16] Kubo H et al 2002 Phys. Plasmas 9 2127-33

[17] Loarte A et al 2002 Plasma Phys. Control. Fusion 44 1815-44

[18] Ehrenberg J K 1995 JET-P(95)18

[19] Koslowski H R et al 2001 Proc. 28th EPS Conf. on Controlled Fusion and Plasma Physics (Madeira, 2001) paper P3.010

[20] Saibene G et al 2002 Plasma Phys. Control. Fusion 44 1769-99

[21] Hillis D L et al 2001 43rd Annual Meeting of the Division of Plasma Physics of the American Physical Society GP1.50 